\title{
XXVIII Evolution by subtraction
}

\section{Rev. F.H. Hummel M.A.}

To cite this article: Rev. F.H. Hummel M.A. (1880) XXVIII Evolution by subtraction, Philosophical Magazine Series 5, 10:61, 190-199, DOI: 10.1080/14786448008626916

To link to this article: http://dx.doi.org/10.1080/14786448008626916

$$
\text { 曲 Published online: } 08 \text { May } 2009 .
$$

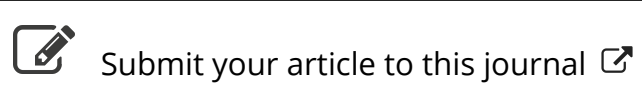

\footnotetext{
山 Article views: 2
}

Q View related articles $\sqsubset$ 
a number of open currents, which render the gas incandescent in their path, and reach so much the further the greater the exhaustion is. If, when the exhaustion is not very great, the length of the discharge issuing from the kathode is less than the interval between the kathode and the next point of discharge (from which the first positive layer issues), there then must exist between the kathode-light and the first positive layer a space traversed by no current, in which therefore there is no luminosity, the so-called " dark space."

If the current-length of the kathode-discharge increases in consequence of increased exhaustion, so that it is equal to the interval between the kathode and the next point of discharge, then the kathode-rays reach the positive light and the dark space vanishes. If the length of the kathode-current become still greater than that interval, then the kathode-light advances into the space into which also flows a current from the second point of discharge, and the kathode-light penetrates into the positive light. In exactly the same way is explained the production of the dark space between each bundle of secondary negative light and the layer which follows it, and the dark spaces which the layers show between them at comparatively small exhaustion, whilst they are in contact when the exhaustion is greater.

In the same way the phenomena not to be explained by previous views, those obtained (pp. 178-183) with kathodes of different forms and positions, contain nothing more that is puzzling; and there is no more need to suppose a to-and-fro motion of electricity, or of a repeated zigzag path, nor of a new and non-luminous mode of discharge.

[To be continned.]

XXVIII. Evolution by Subtraction.

By the Rev. F. H. Hummel, M.A.*

"WHEN we require to find a root (say, e.g., the square root) of an arithmotical quantity, we begin by 'pointing,' i. e. dividing off the digits of our given quantity into equal groups, starting from the units' place, each group containing a number of digits equal to the index of the required root; the last group on the left containing so many digits as happen to remain to it. On this last group we commence operations, finding the square root of the square number next below it in numerical value. The remaining steps are simply successive solutions of a continually recurring equation of the form $n^{2}=a^{2}+2 a x+x^{2}$; in which, by approximating to $x$ at each step, and then adding its approximate value to $a$ for the next

* Communicated by the Author. 
equation, we arrive at a value, actual or approximate, for $n$, the required root. For the cube root, the corresponding equation is considerably more involved; and beyond this point our authors abandon the method.

"Let us examine this traditional method of evolving square and cube roots. The second and following steps are methodical enough, being in fact solutions of a known equation, and lead surely to correct results. But the first step depends entirely on an exercise of memory; it is not performed by method, but by an arbitrary process of recalling previous knowledge; it is, in a word, guesswork. Take, for instance, the square root of 6561 ; having given that the first figure of the result is 8 , the rest follows of course; but how do we know the first figure? Or suppose the given square were 64 simply; our text-books furnish absolutely no mothod whatever for finding the square root of a number of less than three digits.

"Yet there is a method, not mentioned in our books, a method of singular, almost ridiculous, simplicity, that will evolve with certainty the square root of any exact square whatever. The rule is no more than this:- Subtract successively the even numbers; the last remainder will be the square root. Here, for, example, is the square root of 64 above mentioned, evolved by an unerring rule. The process of subtraction has been continued until it left us the required root, and could not then be carried any further.

$$
\begin{aligned}
& n^{2}=64 \\
& \frac{2}{62} \\
& \frac{4}{58} \\
& \frac{6}{52} \\
& 52 \\
& \frac{8}{44} \\
& 10 \\
& \overline{34} \\
& 12 \\
& 22 \\
& n=\frac{14}{8}
\end{aligned}
$$

"Now let us turn to the cube root, and take this simple rule:-For the nth subtrahend, multiply $\mathrm{n}$ by 6 , and add the previous subtrahend. The last remainder will be the cube root. Thus the first subtrahend is 6 ; the next, $6 \times 2+6=18$; the third, $18+18=36$; then $24+36=60$; and so on. The series 
of subtrahends is, in fact, $6 \times$ the series of 'triangular' numbers, $1,3,6,10$, \&c. Here is the cube root of 729 found by this process.

$$
\begin{aligned}
& n^{3}=729 \\
& \frac{6}{723} \\
& \frac{18}{705} \\
& \frac{36}{669} \\
& \frac{60}{609} \\
& \frac{90}{519} \\
& 126 \\
& \overline{393} \\
& 168 \\
& n=\begin{array}{r}
\overline{225} \\
\frac{216}{9}
\end{array}
\end{aligned}
$$

"It is clear that similar rules may be framed for finding the roots of higher powers."

Considering this method of evolution to be of great importance, and believing it (under correction) to be wholly novel, I must at once assign the credit of its invention to the real author. To the foregoing portion of this paper I have contributed nothing but the wording; the matter of it was communicated to me by my esteemed friend and neighbour the Rev. W. B. Cole, of Shanklin. In the belief that the method is altogether new to the world (though its author seems to have retained it in petto for some years), I have ventured to give it the title of "Cole's Method of Evolution;" and with his permission I now present it to the public, with a few remarks of my own on its more extended application.

The first point to which I shall refer is the principle on which the method is based, so that we may obtain a general formula whence to frame rules of evolution for the fourth, fifth, or any higher roots. We shall then be in a position to decide on the extent of its application, and to frame tables for its employment.

Having given a known power of an unknown number (call it $n^{m}$ ), we subtract successively the terms of a series, and find for a last remainder the required root $n$. Obviously this series is the one whose sum to $n$ terms $=n^{m}-n$; and obviously, 
again, this series is the one whose general term

$$
u_{n}=\left(n^{m}-n\right)-\left\{(n-1)^{n}-(n-1)\right\} \text {. }
$$

If we substitute $1,2,3$, \&c. for $n$ in this formula, we shall obtain the successive subtrahends for any given index $m$.

No confusion need be apprehended from the double sense in which $I$ am using the symbol $n$, to indicate both the required root and the ordinal number of each term in the series; for during the operation the value of the root is unknown to us, and we substitute the integers successively in the hope of finding it; and a consideration of the series will show that the number of terms required is in every case $=n$, including the zero term for $n=1$.

The general term as given above is not yet in a form adapted for simple arithmetical calculation, except for very low values both of $m$ and $n$; but by expansion we may cast it into a form capable of resolution into factors in such a way as to make the calculation easier. For

$$
\left(n^{m}-n\right)-\left\{(n-1)^{m}-(n-1)\right\}=n^{m}-1-(n-1)^{m} ;
$$

which, expanded by the Binomial Theorem, the highest powers of $n$ cancelling, leaves us

$$
u_{n}=-1+m n^{m-1}-\frac{1}{2} m(m-1) n^{m-2}+\& c, \pm m n \mp 1 .
$$

Here, if $m$ be even, the last term is -1 , and the expression for

$$
\begin{aligned}
u_{n}=2\left\{-1+\frac{1}{8} m n\left(n^{m-2}-\frac{1}{2}(m-1)\right.\right. & n^{m-3}+\& \mathrm{c} . \\
& \left.\left.-\frac{1}{2}(m-1) n+1\right)\right\} ;
\end{aligned}
$$

if $m$ be odd, the last term is +1 , which cancels, and the expression becomes

$$
u_{n}=m n\left(n^{m-2}-\frac{1}{2}(m-1) n^{m-3}+\& c .+\frac{1}{2}(m-1) n-1\right) .
$$

In this latter case the quantity within the bracket will always be divisible by $(n-1)$, as may be seen by an inspection of the coefficients. So far, then, the method appears to favour the odd indices, which might have been expected to present the greatest difficulty; and an even index, if not a power of 2 , may be reduced to an odd one by one or more operations for the square root.

But an alternative rule offers itself, of making each term of the subtrahend series to be itself the sum of a series. Such a series will of course be that of the differences of successive subtrahends; and in working we shall have to add to each term the last preceding subtrahend, which will have been the sum of all the previous terms. The general term of this series will be $u_{n+1}-u_{n}$. Now this expression

$$
\begin{aligned}
& =(n+1)^{m}-1-n^{m}-\left\{n^{m}-1-(n-1)^{m}\right\} \\
& =(n+1)^{m}+(n-1)^{m}-2 n^{m} .
\end{aligned}
$$

Phil. Mag. S. 5. Vol. 10. No. 61. Sept. 1880. 
The first term of this addition is cancelled by the $-2 n^{n^{2}}$; the expression will accordingly be the double of the positive terms of the expansion of $(n-1)^{m}$, beginning at the third term. If $m$ is even, the last term will be +2 . Therefore we may write as an alternative formula,

$$
\begin{array}{r}
u_{n+1}-u_{n}=m(m-1) n^{m-2}+\underbrace{\frac{\sum^{m}}{m-4}} \cdot \frac{n^{m-4}}{3.4}+\frac{\sum^{m}}{\underbrace{}_{-6}-6} \cdot \frac{n^{m-6}}{3.4 .5 .6} \\
+\& c .[+2 \text { if } m \text { is even }] .
\end{array}
$$

All these coefficients are integral. When $m$ is even, they will recur in reverse order, like those of the Binomial Theorem, and the whole expression will be divisible by $n^{2}$, with the final 2 for remainder. When $m$ is odd, the whole expression will be divisible by $n$; and when $m$ is prime, it will also be divisible by $m$. When $m-1$ is prime, it will be divisible by $(m-1) n^{2}$ with remainder 2 . When $m$ is even but not a multiple of 4 , it will be divisible also by $\left(n^{2}+1\right)$ with remainder 2 .

We have thus for any integral value of $m$, whether odd or even, two alternative formula, in either of which, by substituting $1,2,3$, \&c. successively for $n$, we may obtain the terms of the required series, either directly, or by summing an ancillary series; and by continuously subtracting these terms in their order from the given quantity $n^{m}$, we should at last find $n$ as a last remainder, at a point beyond which no further subtraction is possible, since every term of the series is greater than $n$. As both these formulæ depend upon the value of $m$, it will be possible to establish by means of them an arithmetical rule of evolution for an $y$ positive integral index whatever; it becomes simply a question of ingenuity in each case to cast one or other of the formulæ into the most convenient shape for working.

The rule already given for the square root may be derived directly from the recognition of $n^{2}-n=n(n-1)$ as double the sum to $n$ terms of the series of consecutive integers; but by our formulæ $u_{n+1}=2 n$; or $u_{n+1}-u_{n}=2$, the difference of successive even numbers. So for the cube root, $u_{n+1}-u_{n}=6 n$, or $u_{n+1}=3 n(n+1)$-formulæ which correspond to the two forms of the rule as stated above. In like manner we may find a rule for extracting the 4th root thus: for the $(n+1)$ th subtrahend, multiply $n^{2}$ by 12 , and add $2+$ the previous subtrahend; for if $m=4, u_{n+1}-u_{n}=12 n^{2}+2$.

Now we are well outside the pale of the old rules, which stopped short at the cube root. I propose to venture a few steps into the unexplored region, and find series and rules for some of the higher indices by means of the formulæ already established. Taking the integers in order for values of $m$, and working out their series, we may discover from the lower 
values some further properties which may reduce the labour of calculation for the higher values.

Let us begin by working out a sum in the 5th root. $E x$. Find the 5th root of 1048576 . Here $n=5$.

$\therefore u_{n+1}-u_{n}=20 n^{3}+10 n=10 n\left(2 n^{2}+1\right)=10 n\left\{n^{2}+\left(n^{2}+1\right)\right\}$. Hence the Rule:-Cut off the final digit of the given number, and commence the subtraction at the tens' digit. For tho $(n+1)$ th subtrahend, add to $n^{2}$ the next higher number, multiply the sum by $n$, and add to the product the previous sulbtrahend. Replace the final digit to the last remainder.

\begin{tabular}{|c|c|c|c|c|}
\hline $\begin{array}{r}1^{2}=1 \\
+2\end{array}$ & $\begin{array}{r}2^{2}=4 \\
+5\end{array}$ & $\begin{array}{r}3^{2}=9 \\
10\end{array}$ & $\begin{aligned} 4^{2}= & 16 \\
& 17\end{aligned}$ & $n^{5}=104857$ \\
\hline \multirow{5}{*}{-} & $\overline{9}$ & $\overline{19}$ & $\overline{33}$ & $\overline{104854}$ \\
\hline & $\times 2$ & 3 & 4 & $\begin{array}{r}1000 \pm \\
21\end{array}$ \\
\hline & $\begin{array}{r}\overline{18} \\
+3\end{array}$ & $\begin{array}{l}57 \\
21\end{array}$ & $\begin{array}{r}132 \\
78\end{array}$ & $\begin{array}{r}\overline{104833} \\
78\end{array}$ \\
\hline & 21 & $\overline{78}$ & 210 & $\overline{104755}$ \\
\hline & - & - & - & 210 \\
\hline $\begin{array}{r}5^{2}=25 \\
26\end{array}$ & $\begin{array}{r}6^{2}=36 \\
37\end{array}$ & $\begin{array}{r}7^{2}=49 \\
50\end{array}$ & $8^{2}=64$ & $\begin{array}{r}104545 \\
465\end{array}$ \\
\hline 51 & $\begin{array}{r}73 \\
6\end{array}$ & $\overline{99}$ & $\begin{array}{r}129 \\
8\end{array}$ & $\overline{104080}$ \\
\hline $\begin{array}{l}255 \\
210\end{array}$ & $\begin{array}{l}438 \\
465\end{array}$ & $\begin{array}{l}693 \\
903\end{array}$ & $\begin{array}{l}1032 \\
1596\end{array}$ & $\overline{103177}$ \\
\hline & & & & 1596 \\
\hline 465 & $\underline{903}$ & 1596 & 2628 & $\overline{101581}$ \\
\hline & & & & 2628 \\
\hline $9^{2}=81$ & $\begin{aligned} & 10^{2}=100 \\
& 101\end{aligned}$ & $11^{2}=121$ & $12^{2}=144$ & 98953 \\
\hline & & & & 4095 \\
\hline $\begin{array}{r}163 \\
9\end{array}$ & $\begin{array}{r}201 \\
10\end{array}$ & $\begin{array}{r}243 \\
11\end{array}$ & $\begin{array}{r}289 \\
12\end{array}$ & 94858 \\
\hline & & & & 6105 \\
\hline $\begin{array}{l}1467 \\
2628\end{array}$ & $\begin{array}{l}2010 \\
4095\end{array}$ & $\begin{array}{l}2673 \\
6105\end{array}$ & $\begin{array}{l}3468 \\
8778\end{array}$ & 88753 \\
\hline & & & & 8778 \\
\hline 4095 & 6105 & 8778 & 12246 & $\overline{79975}$ \\
\hline & & & & 12246 \\
\hline $15^{2}=169$ & $14^{2}=196$ & $15^{2}=225$ & & $\overline{67729}$ \\
\hline & 197 & 226 & & 16653 \\
\hline 339 & 393 & 451 & & 51076 \\
\hline 13 & 14 & 15 & & 22155 \\
\hline $\begin{array}{r}4407 \\
12246\end{array}$ & $\begin{array}{r}5502 \\
16653\end{array}$ & $\begin{array}{r}6765 \\
22155\end{array}$ & & 28921 \\
\hline & & & & 28920 \\
\hline 16653 & 22155 & 28920 & & \\
\hline & & & & 6 \\
\hline
\end{tabular}


[Incidentally we may note that this rule for the 5th root affords an easy proof of the arithmetical proposition that the final digit of any number is the same as that of its 5th power, and consequently of any $(4 p+1)$ th power, $p$ being any positive integer.]

I have given this example worked out, with the marginal calculations, to show what such a sum in this method will look like, and to demonstrate the facility of the working. I shall content myself with subjoining rules for the $(n+1)$ th subtrahend for each of the next half-dozen series.

6 th root. $u_{n+1}-u_{n}=30 n^{2}\left(n^{2}+1\right)+2$. Rule:-multiply $n^{2}$ by the next higher number, and again by 30 , and add $2+$ the previous subtrahend.

7 th root. $u_{n+1}=7 n(n+1)\{n(n+1)+1\}^{2}$. Take the product of $n$ and $(n+1)$, multiply it by the square of the next higher number, and again by 7 . But for the higher values of $n$ the trouble of squaring will lead us to prefer the alternative formula, $u_{n+1}-u_{n}=7 n\left\{6 n^{2}\left(n^{2}+1\right)+4 n^{2}+2\right\}$; multiply $n^{2}$ by the next higher number, and again by 6 ; add to the product 4 times $n^{2}$ and 2 ; multiply the sum by $n$, and again by 7 , and add the previous subtrahend.

8th root. $u_{n+1}-u_{n}^{n}=28 n^{2}\left(n^{2}+2\right)\left\{n^{2}+\left(n^{2}+1\right)\right\}+2$. Add to $n^{2}$ the next higher number, multiply the sum by $\left(n^{2}+2\right)$, and again by $n^{2}$, and again by 28 , and add $2+$ the previous subtrahend.

9 th root. $u_{n+1}=3 n(n+1)\left\{3\left(n^{2}+n+1\right)^{3}+n^{2}(n+1)^{2}\right\} ;$ a formula which will be more convenient for higher terms in the shape

$$
\begin{array}{r}
3 n(n+1)[n(n+1)\{3[\{n(n+1)+1\}\{n(n+1)+2\}+1] \\
+n(n+1)\}+3] .
\end{array}
$$

Find the product of $n(n+1)$; take the product of the next two consecutive numbers above it, add 1 , multiply by 3 , add $n(n+1)$; multiply the sum by $n(n+1)$, add 3 , and multiply by $3 n(n+1)$.

10 th root.

$$
u_{n+1}-u_{n}=30 n^{2}\left(n^{2}+1\right)\left\{3 n^{2}\left(n^{2}+3\right)+n^{2}+\left(n^{2}+3\right)\right\}+2 .
$$

Take the product of $n^{2}$ and $\left(n^{2}+3\right)$, multiply by 3 , and add to the product $n^{2}$ and $\left(n^{2}+3\right)$; multiply the sum by $n^{2}$, again by $\left(n^{2}+1\right)$, and again by 30 , and add $2+$ the previous subtrahend.

11 th root.

$$
\begin{array}{r}
u_{n+1}=11 n(n+1)\{n(n+1)+1\}[n(n+1)\{n(n+1)+1\} \\
\{n(n+1)+3\}+1] .
\end{array}
$$

Take the product of $n(n+1)$, multiply it by the next higher 
number, and again by the next odd number above that; add 1 to the product, and multiply by the product

already found, and again by 11 .

$$
n(n+1)\{n(n+1)+1\}
$$

Let these examples suffice; we have reached a point at which the formula begin to have a complicated appearance, though the practical working of them is not beyond the capacity of a child, and already we have made a great advance on the results of the older methods. We may already perceive from our investigation that the formulæ for $u_{n+1}-u_{n}$ are of most use in simplifying results for the lower indices; but that as we go higher we can more safely rely on the formulæ for $u_{n}$ which more closely follow the Binomial Theorem, and so, yielding readily to division by such factors as $(n+1)$, $\left(n^{2}+n+1\right)$, \&c., often give us combinations of consecutive or nearly consecutive integers, which are far more easily worked in practice than described in rules.

Now let us tabulate the results of the rules already found. I will give ten terms of each series, so that they may be easily verified by finding their sum in each case to be $10^{m}-10$; that is, a row of nines with a zero at the end.

$m=1$. All the terms are zero.

$m=2 . \quad 0+2+4+6+8+10+12+14+16+18+\&$ c.

$m=3 . \quad 0+6+18+36+60+90+126+168+216+270+\& c$.

$m=4 . \quad 0+14+64+174+368+670+1104+1694+2464$ $+3438+\& c$.

$m=5 . \quad 0+30+210+780+2100+4650+9030+15960$ $+26280+40950+\& c$.

$m=6 . \quad 0+62+664+3366+11528+31030+70992+144494$ $+269296+468558+\& c$.

$m=7 . \quad 0+126+2058+14196+61740+201810+543606$ $+1273608+2685816+5217030+\& \mathrm{c}$.

$m=8 . \quad 0+254+6304+58974+325088+1288990+4085184$

$+11012414+26269504+56953278+\& \mathrm{c}$.

$m=9 . \quad 0+510+19170+242460+1690980+8124570$

$+30275910+93864120+253202760+612579510+\& c$.

$m=10.0+1022+58024+989526+8717048+50700550$

$+222009072+791266574+2413042576$

$+6513215598+\& c$.

$m=11.0+2046+175098+4017156+44633820+313968930$

$+1614529686+6612607848+22791125016$

$+68618940390+\& c$. 
Having now the actual numbers before us, we can verify in fact the relation which we may find from the algebraic formulæ to exist between the terms of consecutive series. Denoting the number of a series by a bracketed index, we may write this relation thus : $u_{n}^{(m+1)}=n u_{n}^{(m)}+\Sigma_{n-1}^{(m)}+2(n-1)$; the $n$th term of any series $=n$ times the $n$th term of the preceding series + the sum of the $(n-1)$ previous terms of that series ttwice $(n-1)$. Thus, having given five terms of the 6 th series, the 5 th term of the 7 th series will be

$$
5 \times 11528+62+664+3366+2 \times 4=61740 .
$$

By this formula we may extend our tables to any number of series, and to any number of terms in each.

We may notice in passing the recurrence of final digits of every five terms of each series, repeated in every group of four series, thus :

$$
\begin{aligned}
& m=4 p, \dot{0}, 4,4,4, \dot{8} ; \quad m=4 p+1, \dot{0}, 0,0,0, \dot{0} ; \\
& m=4 p+2, \dot{0}, 2,4,6, \dot{8} ; \quad m=4 p+3, \dot{0}, 6,8,6, \dot{0} .
\end{aligned}
$$

So the sum of $5 p$ or $5 p+1$ terms in every series, and of $5 p-1$ terms of the odd series, have zero for the final digit, as would be necessary for powers of numbers of the form $5 p$ or $5 p \pm 1$. All the terms of each series are even numbers, as we might have foreseen from the algebraic formulæ. This, too, was necessary ; for the powers of odd numbers being all odd, and those of even numbers all even, $n^{m}-n$ is in every case even.

So much, then, for the mode of constructing the subtrahend series. Before proceeding further, I may remark that this method is of course as useful for involution as for evolution; for being in possession of the series whose sum to $n$ terms is $n^{m}-n$, we have only to add that sum to $n$ to obtain the value of $n^{m}$.

Hitherto we have seemed to assume that the number whose $m$ th root is to be taken is the exact $m$ th power of an integral root. But clearly, if this were an indispensable condition for the applicability of the method, its use would be extremely limited. Let us consider the case in which the given number is not an exact $m$ th power; we may write it $n^{m} \pm d$. As the evolution is performed throughout by the process of subtraction, every remainder will differ by the same excess or defect $\pm d$ from what it would have been if the given number had been $n^{m}$ exactly. Accordingly, our last remainder will be $n \pm d$; that is, we have left a number which differs from $n$ by the same excess or defect as that by which the given number differed from $n^{m}$. As this is by far the most usual case, it is evident that the last remainder is not, after all, the right place 
to look even for an approximation to the required root, as a general rule. But our investigation of the series has shown us that $n^{m}-n$ is always the sum of $n$ terms; and consequently, in approximating to the $m$ th root of $n^{m} \pm d$, we can discover $n$ at once by counting the number of terms subtracted. It must be remembered that the first term is in every case zero, and therefore the number of actual subtractions we have performed will be $n-1$.

Having thus found $n$, which is so far an approximation to the required root, we may now obtain a nearer approximation by the help of the last remainder, which we have seen to be $n \pm d$. For we can now determine the value and sign of $d$ by subtracting $n$ from the last remainder, and we know that our given number is $n^{m} \pm d$. There are, of course, known methods by which the $m$ th root of $n^{m} \pm d$ may be pretty accurately found; but, omitting these, let me point out that this method itself will furnish a means of approximating tolerably nearly to the root, by adding to $n$ (or, if the sign of $d$ prove to be negative, subtracting from $n$ ) the quotient of $d$ divided by the next term of the subtrahend series following the last one subtracted. To write it algebraically,

$$
\sqrt[m]{ } /\left(n^{m} \pm d\right)=n \pm \frac{d}{u_{n+1}} \text { nearly. }
$$

For example, let it be required to find the 11th root of 8590035271. After 7 subtractions we arrive at a last remainder 100687. Therefore $7+1=8$ is an approximate root. The next subtrahend would have been 22791125016 ; accordingly we may take as a still closer approximation to the required root

$$
8+\frac{100687-8}{22791125016}=8 \cdot 000044 \text { nearly. }
$$

Our investigation, then, will lead us to a restatement of Cole's method in some such form as this:-

To find the $m$ th root of a given number, find a sufficient number of terms of the series whose sum to $n$ terms is $n^{m}-n$; subtract these terms successively from the given number as far as such subtraction is possible. The number of such subtractions +1 , if it be equal to the last remainder, will be an exact $m$ th root. If not, it will be an approximate $m$ th root; and for a nearer approximation, find the difference between this first approximate root and the last remainder, and add (or if the remainder was the less, subtract) the quotient of that difference divided by that term of the series which follows next after the last term subtracted. 